\title{
Research on Missile Attack and Defense Modeling of High-level Missile Based on Discrete Event
}

\author{
Yao Yu ${ }^{1, \text { a }}$, Zhong-wen Zhao ${ }^{1, b}$, Ya-Shuai Lv ${ }^{1, c}$ and Huang-huang Guo ${ }^{1, d}$ \\ ${ }^{1}$ Science and Technology on Complex Electronic System Simulation Laboratory, Equipment \\ Academy,Beijing,101400,China \\ a799448071@qq.com
}

Keywords: discrete event; state diagram; missile attack and defense; event-driven

\begin{abstract}
The traditional analysis simulations are generally based on the time stepping mechanism in HLA. This method will greatly affect the simulation efficiency due to in a period of time no events occurred. This paper attempts to simulate high-level missile attack and defense using discrete event simulation technology, avoiding the idle phenomenon. This paper modifies the state diagram modeling method's shortcomings, that is, events can not be generated from the activities and activities can not be detailed. According to this method, the radar reconnaissance and interference equipments and missiles in missile attack and defense are simulated by discrete events which prove the effectiveness of solving missile attack and defense problems.
\end{abstract}

\section{Introduction}

High-level missile attack and defense analysis simulation has a high demand to efficiency. This paper uses the discrete event-driven method to improve simulation efficiency. The more common discrete event modeling methods include state diagram method ${ }^{[3] \sim 5]}$, IDEF0 model method ${ }^{[6] ~[7]}$, Petri net ${ }^{[8]}$ ${ }^{\sim 10]}$. These methods are not clear enough and comprehensive currently. Petri net has a greater advantage in a modeling analysis, but it's not conducive to reading in large-scale system modeling and difficult to convert into programs. This paper analyzes the elements of the discrete event system, considering the application scenarios of the missile attack and defense, and modifies the shortcomings of the state diagram modeling method in which events can not be generated from the activities and activities can not be detailed. According to the interactive module of missiles and radar jamming equipment in missile attack and defense, the improved state diagram is used to model the missile attack and defense system. Finally realizing simulation in the ROSS engine, and a case analysis proved that the discrete event simulation can really solve the problem of high-level missile attack and defense.

\section{Discrete Event System Modeling Method}

Analyzing Modeling Factor of Discrete Event System. Before modeling the missile attack and defense by the discrete event modeling method, we must first understand the necessary aspects of the missile attack and defense modeling. we according to the elements of the discrete event system modeling, and it will be modeled from the following five aspects:

Entity. Entities are the physical components of the system, such as missiles, radar reconnaissance and interference equipments.

Property. Property can reflect the nature of the entity, such as the characteristics of text, digital and logical to express, such as the missile interference state belonging to the text property, the coordinates of the missile belonging to the numeric property, etc. Therefore, the property can be understood as a data structure. The property of the modeling process don't not need to be reflected. 
State. The state of the discrete event simulation system is different from the state of the time step simulation. Discrete event simulation is to push the simulation clock according to the processing event, that is, event-driven. That means that before the arrival of another event, the state of the entity remains unchanged. So it reflects the discrete state.

Event. Events can cause changes of the system state, then triggering different activities. Events can also be generated from activities and it is the core element in thw discrete event system. In the missile attack and defense system, all entities can be seen as in the "static" process, that is, the missile is not in the movement. Because of the event trigger, making the missile's properties continue to change, and changing properties reflects the movement of the missile.

Activity.The process of two adjacent events is called activity, and action is generally used to describe a brief operation, which is a behavior that consists of one or more actions. In the missile attack and defense system, Activities constitute the function of the detection, identification, interference and so on. It is because of these activities, each entity has different functions.

The links among five elements. Entity is the package of the process. It is determined by its activities, states, property, different states to perform different activities. The execution of the activity changes the property value of the entity. The change of the property value reflects the movement of the "static" entity. Events play a trigger role in the whole process, because the change of the state is caused by the event. Entities receive different events in different states, perform different activities, generate new events, which may act on their own entities, and may also act on other entities, causing changes in the entity's state, performing corresponding activities, and thus forming Loop, push the simulation.

Improved state diagram modeling method. A state diagram is a good way to solve event-driven modeling, which describes the dynamic behavior of an entity caused by an event-driven process that allows a system or entity to transition from the current state to another. The state transition of the state diagram is mainly composed of five parts: the source state, the trigger event, the guard condition, the action / activity and the target state, as shown in Figure 1.

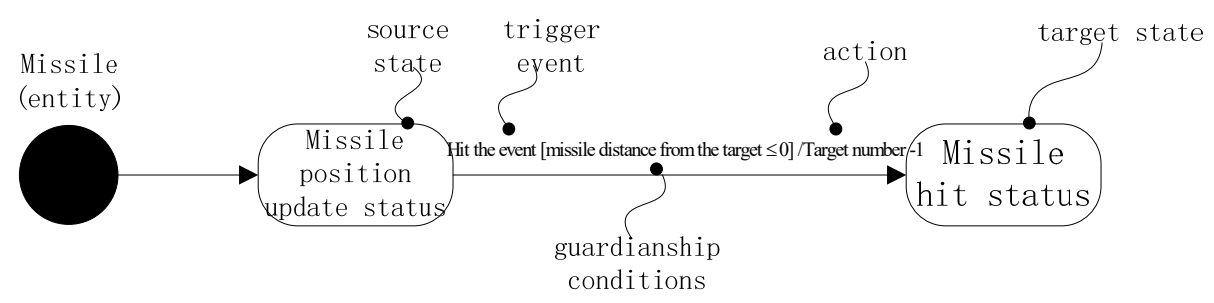

Figure 1 The illustration of the state transition based on state diagram

Among them, when the trigger event to meet the guardianship conditions, the system implement the corresponding actions or activities in order to realize the source state to target state conversion. The state diagram can express the three elements of the entity, state and activity of the modeling elements of the discrete event system, but still need to be further improved for the event expression. State diagram can express that events cause entity state changes, but do not satisfy discrete events that can either trigger activity or can be generated from activity due to activities of state diagrams that can not be well expressed or highlighted.

So we highlight the role of triggering events and activities, it reflecting the state of the transfer process more clearly, so as to make up for the disadvantages of the state diagram, the process of improved state diagram shown in Figure 2. The entity in Figure 2 generates the different state caused by the different event, performs the corresponding action activity, and generates a new event from the activity in that state, after event discrimination. 


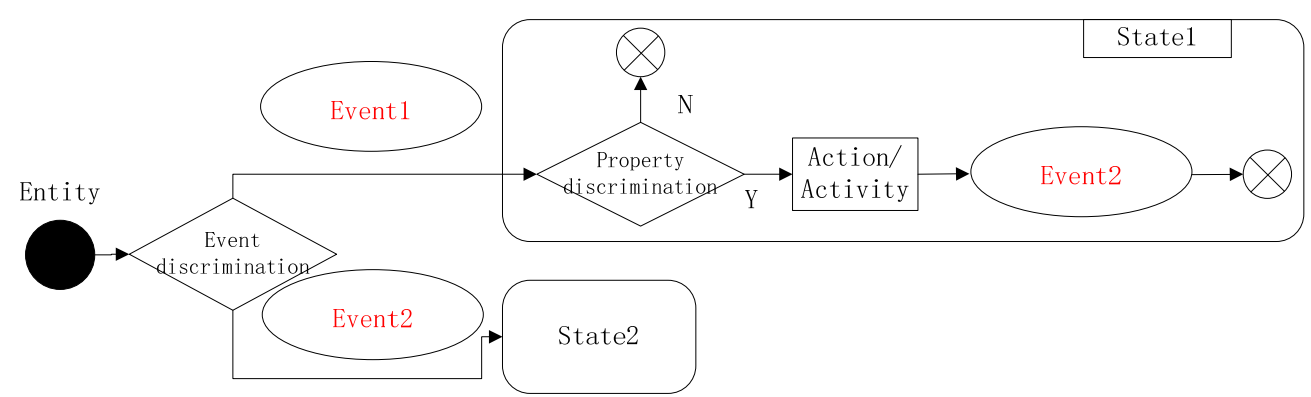

Figure 2 Schematic diagram of discrete event modeling using improved state diagrams The improved state diagram program is implemented as follows:



\section{Missile Attack and Defense Modeling Based on Discrete Event Radar Countermeasure}

The missile attack and defense are generally divided into offensive and defensive sides. The attacking parties generally use a large number of electronic reconnaissance, interference equipments and hard killer weapons to form an attack system, such as a number of aircrafts with long-distance interfere, ground radar interference stations, reconnaissance stations, and aircraft attack formations and missile attack groups, etc. Defense side will organize the defense system, such as ground radar confrontation group and interference group.

This article explores the possibility of discrete event simulation to solve missile attack and defense problems, so we only model missile entities and radar reconnaissance and interference equipment in this section. The module interaction relationship between radar interference equipment and missile is shown in Figure 3. The whole process involves four modules as follows:

The module of radar reconnaissance :When the target enters the reconnaissance range, the target is filtered by coarse filtering, filtering out the point that does not meet the reconnaissance conditions (not satisfied with the radar working medium, frequency band, pulse width, repetition frequency), forming the test probability, and finally using the Monte Carlo method to test targets. If the test fails, the missile will continue to fly into the next round of radar reconnaissance. If the test is successful, the radar reconnaissance and interference the implementation performs the missile interference decision.

The module of missile interference decision:According to the information obtained by the threat of matching and threat library, if the match is successful, then the module directly assigns the missile interference resources to the missiles. If the match fails, according to the experience of threat, and then the module assigns the missile interference resources.

The module of missile jamming effect:This module divides a variety of missile interference into three kinds of interference effects, induced, suppressed and dragged interference effects. If the interference succeeds, missile is disabled. If the interference fails, the missile will not continue to fly.

The module of controlling the moving: This module constantly calculates the missiles' positions. According to the distance between the missile and the target, if the distance is less than or 
equal to 0 , then the missile hit the target. If the distance is greater than 0 , then the missiles continue to fly, and update their own positions.



Figure 3 The diagram of the module interaction relationship between radar interference equipment and missile

According to Figure 3 and the improved state diagram for discrete event modeling, missile entity construction is shown in Figure 4. The missile entity has three status, the operating state (the status of missile position update status), the missile disturbed successfully or hitting (missile failure state), the status of missile interference is in progress. The missile state is determined by the event received, so the missile entity needs event discrimination to determine which state of the implementation of the activities. When receiving the event of missile position update, the missile entity implements the event of missile position update state. If the missile position and target distance is less than or equal to 0 , the missile hits, resulting in a event of missile hit event, then the state of the missile into a missile failure state. If the missile position and target are greater than 0 , the missile is considered to be running, to produce a event of missile position report and a event of missile position update respectively, which will cause the target entity to perform a reconnaissance state. The event of missile position update will cause the missile entity to re-enter the status of missile position update, turning on the next round.

When receiving the event of missile interference, the missile entity enters the status of interference and performs the corresponding activities. If the interference fails, missile entity generates a event of missile position update. If the interference is successful, missile entity enters the status of missile failure.

According to Figure 3 and the improved state diagram for discrete event modeling, missile entity construction is shown in Figure 4. The missile entity has three status, the operating state (the status of missile position update status), the missile disturbed successfully or hitting (missile failure state), the status of missile interference is in progress. The missile state is determined by the event received, so the missile entity needs event discrimination to determine which state of the implementation of the activities. When receiving the event of missile position update, the missile entity implements the event of missile position update state. If the missile position and target distance is less than or equal to 0 , the missile hits, resulting in a event of missile hit event, then the state of the missile into a missile failure state ; If the missile position and target are greater than 0 , the missile is considered to be running, to produce a event of missile position report and a event of missile position update respectively, which will cause the target entity to perform a reconnaissance state. The event of missile position update will cause the missile entity to re-enter the status of missile position update, turning on the next round.

When receiving the event of missile interference, the missile entity enters the status of interference and performs the corresponding activities. If the interference fails, missile entity 
generates a event of missile position update. If the interference is successful, missile entity enters the status of missile failure.

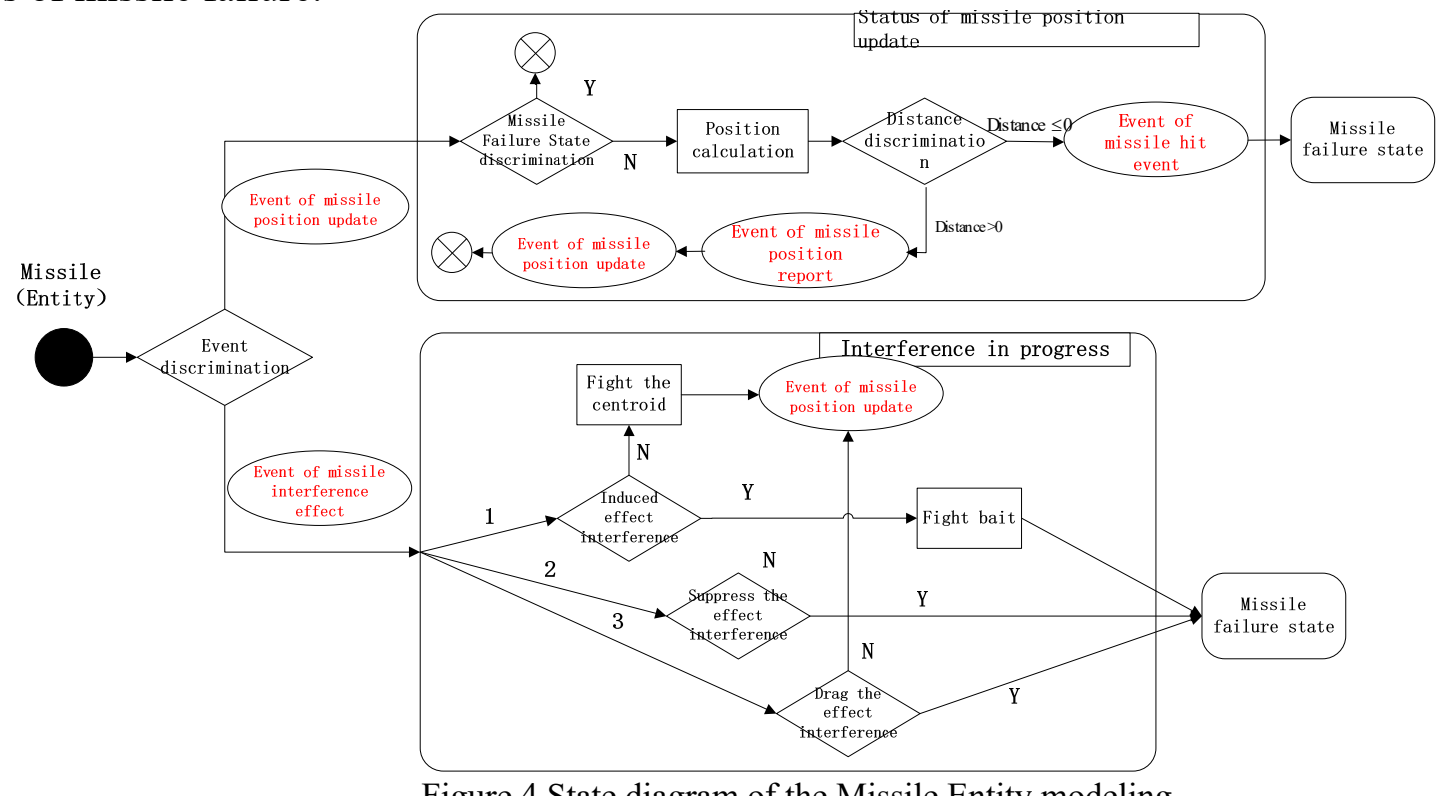

Figure 4 State diagram of the Missile Entity modeling

For the construction of the target entity as shown in Figure 5, when the missile entity receives the event of missile position report, the target entities enter the status of reconnaissance, implementing corresponding activities. After the successful detection of missile targets, the target entity generates the event of interference decision. When the interference decision event is received, the target entity enters the interference decision state, performing the interference resource configuration, generating the missile interference event, and sending the event to the missile entity. The missile entity receiving the event of missile hit, enters the status of missile hit, and performs the statistics of missile hit.



Figure 5 State diagram of the Target Entity modeling

\section{Simulation experiment and case analysis}

In the Ubuntu Kylin 15.10 system, we set up a discrete event simulation model of missile attack and defense based on the ROSS simulation engine after clarifying the state definition and conversion logic of each module. We design and initialize the parameters of the interface, the use of $\mathrm{C}$ language programming to realize each module.

We need to carry out missile attacks on 128 targets distributed by the enemy, each with a radar and interference device. The missile is fired at the center of the target group at $1000 \mathrm{~km}$. The missile flight speed is $306 \mathrm{~m} / \mathrm{s}$. The radar power is $10 \mathrm{kw}$. The radar antenna gain is 6 . The operating frequency is $9.7 \mathrm{GHz}$. The wavelength $\mathrm{K}=0.05 \mathrm{~m}$. The receiver sensitivity $\mathrm{S}=10^{-7} \mathrm{~W}$. Missile interference resources can be recycled and the number of resources is 30 . The loss factor are not considered. Due to terrain restrictions, each firing up to 120 missiles, the enemy's resources re-filled, we explore how many missiles can complete the missile raid task. 
First destroying all targets at once without the limits of terrain restrictions, the relationship between the number of missiles and target hit is shown in Figure 7(a). From this figure, the missile hit rate increases as the number of missiles increases on the whole. However, when the number of missiles is in the 90-170, the missile hit rates are not obvious as the number missiles increase. In order to improve the hit rate, the first hit can launch 90 missiles. For the remaining 95 goals, our analysis is the same as above. When the number of missiles is 120 , the missile hit rate is the most highest as shown in Figure 7(b). The last remaining 44 goals require 92 missiles, the last simulation program results are shown in Figure 7(c).

So the whole program we hit three times and the number of each hit was 90, 120 and 92.

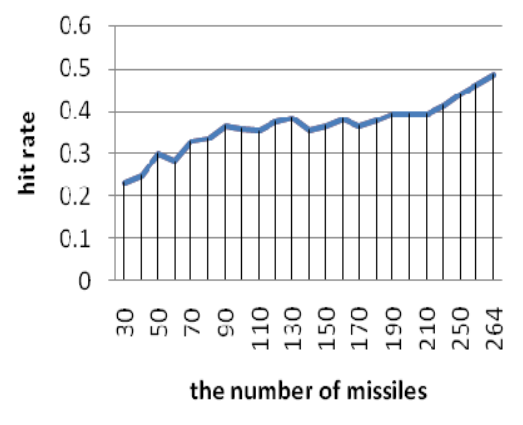

(a)

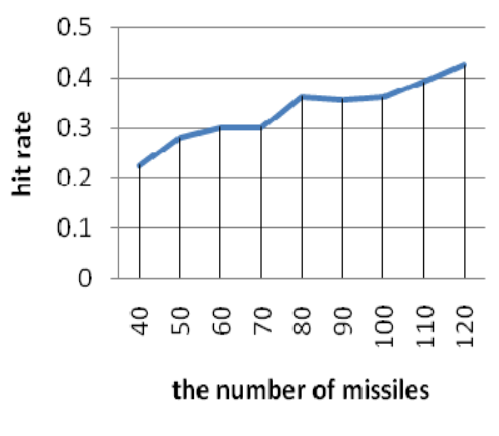

(b)

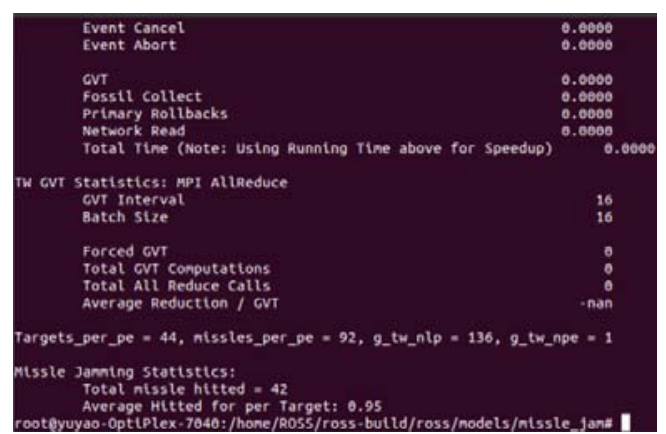

(c)

Figure 6 The simulation data line graphs and simulation results report interface of the missiles attack and defence

\section{References}

[1] LIU Zhou, ZHU Qi-Dan, ZHU Wei, et al.Study on Object-oriented Petri Net in Naval Gun Weapon System Modeling [J]. Journal of System Simulation, 2005, 17 (6): 1343-1346.

[2] Wei Yong, Xu Tingxue, Pang Dapeng.Study on Modeling and Simulation of Warfare Integrity of Naval Gun Equipment Based on Task [J]. Journal of Artillery Launch and Control, 2010 (4): 15-19.

[3] Zhou Xingjiu. BOM-based parallel discrete event simulation modeling technology research and implementation [D]. National Defense University of Science and Technology, 2010.

[4] Yu Cun, Huang Lijun, Huang Haoran, et al.Analysis and Implementation of IEC104 Protocol Based on UML Statechart and Qt State Machine Framework [J]. Power System Protection and Control, 2015 (15): 118-125.

[5] Jiang Jinlong. UML and Petri net modeling method and its application in C $~ 4$ ISR system [D]. Nanjing University of Science and Technology, 2007.

[6] Wang Zhijian, Cai Zixing.Study on Indirect Modeling Method of Petri Net Based on IDEF0 Model [J]. Journal of System Simulation, 2008,20 (15): 3915-3919.

[7] ZHANG Xiao-dong, LIAO Jing-jing, HUANG Xiang-zhao.Research on process modeling of carrier-based dispatching process based on event [J]. China Ship Research, 2014, 9 (6): 1-7.

[8] Wang Sucheng. Performance evaluation of discrete event system based on color Petri net [D]. Anhui University of Science and Technology, 2016.

[9] Luo Xueshan. Discrete Event System Modeling and Simulation Environment (OPMSE) Based on Object Petri Net [J]. Computer Simulation, 2000, 17 (3): 42-44.

[10] Li Jie, Wang Shuting, Chen Liping. Simulation Modeling of Discrete Event System Based on Object-oriented Petri Net [J]. Journal of Huazhong University of Science and Technology (Natural Science Edition), 2001, 29 (5): 12-13. 